\title{
Formal Modeling and Analysis of the OGDC Wireless Sensor Network Algorithm in Real-Time Maude
}

\author{
Peter Csaba Ölveczky and Stian Thorvaldsen \\ Department of Informatics, University of Oslo \\ peterol@ifi.uio.no, stianth@ifi.uio.no
}

\begin{abstract}
This paper describes the application of Real-Time Maude to the formal specification, simulation, and further formal analysis of the sophisticated state-of-the-art OGDC wireless sensor network algorithm. Wireless sensor networks in general, and the OGDC algorithm in particular, pose many challenges to their formal specification and analysis, including novel communication forms, treatment of geographic areas, time-dependent and probabilistic features, and the need to analyze both correctness and performance. Real-Time Maude extends the rewriting logic tool Maude to support formal specification and analysis of objectbased real-time systems. This paper explains how we formally specified OGDC in Real-Time Maude, how we could simulate our specification to perform all the analyses done by the algorithm developers using the network simulation tool ns-2, and how we could perform further formal analyses which are beyond the capabilities of simulation tools. A remarkable result is that our Real-Time Maude simulations seem to provide a much more accurate estimate of the performance of OGDC than the ns-2 simulations. To the best of our knowledge, this is the first time a formal tool has been applied to an advanced wireless sensor network algorithm.
\end{abstract}

\section{Introduction}

This paper describes the application of Real-Time Maude 1715] to the formal specification, simulation, and further formal analysis of the state-of-the-art optimal geographical density control (OGDC) wireless sensor network algorithm [22. To the best of our knowledge, this work represents the first formal modeling and analysis effort of such a complex wireless sensor network system.

A wireless sensor network (WSN) consists of many small, cheap, and lowpower sensor nodes that use wireless technology (usually radio) to communicate with each other [2. Given the increasing sophistication of WSN algorithms - and the difficulty of modifying an algorithm once the sensor network is deployedthere is a clear need to use formal methods to validate system performance and functionality prior to implementing such algorithms.

In [19] we advocate the use of the language and tool Real-Time Maude [15 17], which extends the rewriting logic-based Maude [3] tool to real-time systems, to formally specify, simulate, and further analyze WSN algorithms. The Real-Time 
Maude specification language emphasizes expressiveness and ease of specification. The data types of a system are defined by equational specifications. Instantaneous transitions are defined by rewrite rules, and time elapse is defined by "tick" rewrite rules. Real-Time Maude supports the specification of distributed object-oriented systems, which is ideal for modeling a network system. The high-performance Real-Time Maude tool provides a range of analysis techniques, including: timed rewriting for simulation purposes; timed search for reachability analysis; and time-bounded linear temporal logic model checking. Real-Time Maude has been used to model and analyze a set of advanced real-time systems, such as large communication protocols [188 and scheduling algorithms [13. Such analysis has found subtle design errors not uncovered during traditional simulation and testing. We argue in [19 that Real-Time Maude's expressive specification formalism, and the ease with which new forms of communication can be defined, should make it ideal to model WSN systems.

Jennifer Hou suggested to us her OGDC algorithm 22. for WSNs as a challenging modeling and analysis task. OGDC is a sophisticated state-of-the-art algorithm that tries to maintain complete sensing coverage of an area for as long as possible by switching nodes on and off. It has been simulated by the algorithm developers Zhang and Hou using the simulation tool ns-2 [124].

The OGDC algorithm is an advanced algorithm whose formal specification, simulation, and analysis pose a set of challenges, including:

1. Modeling - and computing with - spatial entities such as coverage areas, angles, and distances.

2. Modeling broadcast communication with transmission delays and limited transmission range.

3. Modeling time-dependent behavior, such as use of timers, transmission delays, and power consumption.

4. Modeling probabilistic behaviors. For example, sensor nodes volunteer to start with certain probabilities, and different values are supposed to be "random values, drawn from a uniform distribution."

5. Simulating and analyzing systems with hundreds of sensor nodes.

6. Analyzing both correctness and, in particular, performance.

This is indeed a challenging set of modeling and analysis tasks. This paper shows how Real-Time Maude met these challenges. In particular, during simulations of the algorithm, we are able to do in Real-Time Maude all the performance analyses that Zhang and Hou performed using the wireless extension of the network simulation tool ns-2 [12. In addition, we have subjected the algorithm to time-bounded reachability analysis and temporal logic model checking.

By modeling transmission delays (which play a significant role in the definition of the OGDC algorithm), and by comparing our performance measures with the ns-2 simulation results, we found a discrepancy which could be explained by a (minor) weakness in the algorithm if the ns-2 simulations did not take the transmission delays into account 1 To test this hypothesis, we also

\footnotetext{
${ }^{1}$ We have not received information of whether the ns- 2 simulations actually took the transmission delays into account, only that it is likely that they did not.
} 
performed Real-Time Maude simulations without considering transmission delays. The results of these simulations are quite similar to the ns- 2 simulations. It is therefore tempting to conjecture that our original simulations provide a much more accurate estimate of the performance of OGDC than the ns- 2 simulations.

Related work. Our work represents - to the best of our knowledge - the first formal modeling and analysis of such a sophisticated WSN algorithm as OGDC. Some attempts at using formal methods on WSNs have focused on modeling TinyOS using automaton-based formalisms (see, e.g., [5]), or have considered simple diffusion protocols for discovering routing trees [11. Our paper [19] explains related work in more detail. That paper also suggests that Real-Time Maude might be a good candidate for formally modeling WSNs, and shows how certain features of such networks, including locations, distances, and communication can be easily modeled in Real-Time Maude. In contrast, this paper focuses on the OGDC case study: It shows how the general techniques suggested in [19] can be applied to specify OGDC; on how advanced features, such as coverage areas, can be modeled in Real-Time Maude; on additional analysis efforts; and on understanding the relationship between the results obtained by Real-Time Maude simulations and by ns-2 simulations. Lately, there has been some initial efforts applying Real-Time Maude to WSNs elsewhere [6]20].

\section{Real-Time Maude}

A Real-Time Maude timed module specifies a real-time rewrite theory 14 of the form $(\Sigma, E, I R, T R)$, where:

$-(\Sigma, E)$ is a membership equational logic 10 theory with $\Sigma$ a signature 2 and $E$ a set of conditional equations. The theory $(\Sigma, E)$ specifies the system's state space as an algebraic data type. $(\Sigma, E)$ must contain a specification of a sort Time modeling the time domain (which may be dense or discrete).

- IR is a set of labeled conditional instantaneous rewrite rules specifying the system's instantaneous (i.e., zero-time) local transitions, each of which is written $\operatorname{crl}[l]: t \Rightarrow t^{\prime}$ if cond, where $l$ is a label. Such a rule specifies a one-step transition from an instance of $t$ to the corresponding instance of $t^{\prime}$, provided the condition holds. The rules are applied modulo the equations $E 3$

- TR is a set of tick (rewrite) rules, written with syntax

$$
\text { crl }[l]:\{t\} \Rightarrow\left\{t^{\prime}\right\} \text { in time } \tau \text { if cond. }
$$

that model time elapse. $\left\{_{-}\right\}$is a built-in constructor of sort GlobalSystem, and $\tau$ is a term of sort Time that denotes the duration of the rewrite.

\footnotetext{
${ }^{2}$ i.e., $\Sigma$ is a set of declarations of sorts, subsorts, and function symbols (or operators).

${ }^{3} E$ is a union $E^{\prime} \cup A$, where $A$ is a set of equational axioms such as associativity, commutativity, and identity, so that deduction is performed modulo A. Operationally, a term is reduced to its $E^{\prime}$-normal form modulo $A$ before any rewrite rule is applied.
} 
The initial states must be ground terms of sort Globalsystem and must be reducible to terms of the form $\{t\}$ using the equations in the specifications. The form of the tick rules then ensures uniform time elapse in all parts of the system.

In object-oriented Real-Time Maude modules, a class declaration

$$
\text { class } C \text { | att } 1: s_{1}, \ldots, \text { att }_{n}: s_{n} \text {. }
$$

declares a class $C$ with attributes att ${ }_{1}$ to att $t_{n}$ of sorts $s_{1}$ to $s_{n}$. An object of class $C$ in a given state is represented as a term $\left\langle O: C \mid a t t_{1}: v_{a l_{1}}, \ldots, a t t_{n}: v a l_{n}\right\rangle$ where $O$ is the object's identifier, and where $v a l_{1}$ to $v a l_{n}$ are the current values of the attributes $a t t_{1}$ to $a t t_{n}$. In a concurrent object-oriented system, the state, which is usually called a configuration, is a term of the built-in sort Configuration. It has typically the structure of a multiset made up of objects and messages. Multiset union for configurations is denoted by a juxtaposition operator (empty syntax) that is declared associative and commutative, so that rewriting is multiset rewriting supported directly in Real-Time Maude. The dynamic behavior of concurrent object systems is axiomatized by specifying each of its concurrent transition patterns by a rewrite rule. For example, the rule

$$
\begin{aligned}
& r 1 \text { [l] : } \mathrm{m}(\mathrm{O}, \mathrm{w})<0: \mathrm{C} \mid \mathrm{a} 1: \mathrm{x}, \mathrm{a} 2: \mathrm{O}^{\prime}, \mathrm{a} 3: \mathrm{z}>\Rightarrow
\end{aligned}
$$

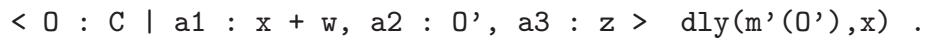

defines a family of transitions in which a message $\mathrm{m}$, with parameters 0 and $\mathrm{w}$, is read and consumed by an object $\mathrm{O}$ of class $\mathrm{C}$. The transitions have the effect of altering the attribute a1 of the object 0 and of sending a new message m' $\left(O^{\prime}\right.$ ) with delay $\mathrm{x}$ (see [17). "Irrelevant" attributes (such as a3, and the right-hand side occurrence of a2) need not be mentioned in a rule.

Timed modules are executable under reasonable assumptions, and Real-Time Maude provides a spectrum of analysis capabilities. We summarize below the Real-Time Maude analysis commands used in our case study.

Real-Time Maude's timed "fair" rewrite command simulates one behavior of the system up to a certain duration. It is written with syntax

(tfrew $t$ in time $<=\tau$.)

where $t$ is the initial state and $\tau$ is a ground term of sort Time.

Real-Time Maude's timed search command uses a breadth-first strategy to search for states that are reachable from a given initial state $t$ within time $\tau$, match a search pattern, and satisfy a search condition. The command which searches for one state satisfying the search criteria has syntax

\section{(tsearch [1] $t=>*$ pattern such that cond in time $<=\tau$.)}

Real-Time Maude also extends Maude's linear temporal logic model checker [3] to check whether each behavior "up to a certain time," as explained in [17], satisfies a temporal logic formula. State propositions are terms of sort Prop, and their semantics should be given by (possibly conditional) equations of the form 
$\{$ statePattern $\} \quad \mid=$ prop $=b$

for $b$ a term of sort Bool, which defines the state proposition prop to hold in all states $\{t\}$ where $\{t\} \quad \mid=$ prop evaluates to true. A temporal logic formula is constructed by state propositions and temporal logic operators such as True, False, (negation), ハ, \/, -> (implication), [] ("always"), <> ("eventually"), and U ("until"). The time-bounded model checking command has syntax

(mc $t$ |=t formula in time $<=\tau$.)

for $t$ the initial state and formula the temporal logic formula.

Finally, the find latest command finds how long it takes, in the worst case, to reach a desired state.

\section{Overview of the OGDC Algorithm}

In a two-dimensional plane, a node with sensing range $r_{s}$ can sense events in a circular coverage area with radius $r_{s}$. It is desirable that the coverage areas of the active nodes cover the entire area to be monitored (the "sensing area") for as long as possible. A large number of nodes is often deployed to extend the lifetime of a wireless sensor network, so that some nodes can be intentionally "put to sleep" to save power. A node that is inactive can be switched on when needed. The process of periodically choosing the nodes that can be put to sleep while maintaining coverage (and connectivity) of the sensing area is called the density control process. The OGDC algorithm [22] is a state-of-the-art density control algorithm, developed by Zhang and Hou, that tries to select the set of active nodes such that their coverage areas provide the minimum amount of overlap.

The network lifetime is divided into rounds, where each round is divided into a node selection phase and a steady state phase. The node selection phase begins with each node having status "undecided" and probabilistically choosing whether or not to volunteer to be a starting node. Each node that volunteers sets its backoff timer to a small value. The node then becomes active when its backoff timer expires, and broadcasts a power-on message which contains the location of the node and a random direction. When an "undecided" node receives a poweron message, it checks if its entire coverage area is covered by the surrounding active nodes, in which case the node becomes inactive. Otherwise, it sets its backoff timer depending on how close the node is to the optimal position w.r.t. the nodes that are currently active. The timer value is set to a gradually larger value as the distance increases and the direction deviates. When the backoff timer of a node expires, the node becomes active and broadcasts a power-on message that may cause other nodes to reset their backoff timers or to become inactive. The network enters the steady state phase when each node is either active or inactive. When a round is over, the density control process starts over again. 


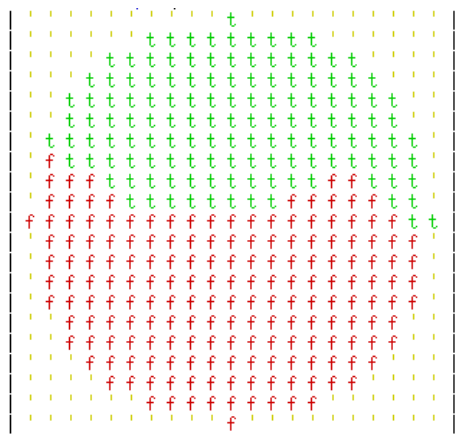

Fig. 1. The bitmap for a node's coverage area

\section{The Real-Time Maude Specification of OGDC}

This section presents a sample of our specification of the OGDC algorithm 4 General techniques for modeling typical WSN features, such as distances and communication, are described in [19].

\subsection{Modeling Locations}

We can represent a location in the plane as a term $x \cdot y$, for rational numbers $x$ and $y$, of the following sort Location 5

sort Location.

op _._ : Rat Rat $\rightarrow$ Location .

\subsection{Modeling Areas Using Bitmaps}

A significant part of the OGDC algorithm consists of checking whether a node's coverage area is completely covered by the coverage areas of other active nodes, since this determines whether or not a node can be switched off. Zhang and Hou suggest in a preliminary version of 22 to use a bitmap to model a node's coverage area. A coverage area is divided into a grid, and each bit in the bitmap represents the center of a grid square. The Real-Time Maude tool is not a graphical tool, but with proper use of the format operator attribute [3, a bitmap can be given an intuitive appearance as shown in Fig. 1. We define a bitmap as a term of sort Bitmap, which consists of a list of BitList: $\sqrt[6]{6}$, which in itself is a list of Bits. A Bit has one of three values: $t$ if the location of the bit is covered by at least one

\footnotetext{
${ }^{4}$ Our specification is explained in detail in 21. The entire executable Real-Time Maude specification can be found at http://www.ifi.uio.no/RealTimeMaude/OGDC

${ }^{5}$ Underbars in the declarations of operators such as _. - denote the places of arguments for "mix-fix" function symbols.

${ }^{6}$ Each BitList corresponds to a "row" in the bitmap.
} 
other active node, $f$ if the location is not covered, or the bit ' that is used to "pad" the circles as shown in Fig. 1. The sort Bitmap is thus defined as follows:

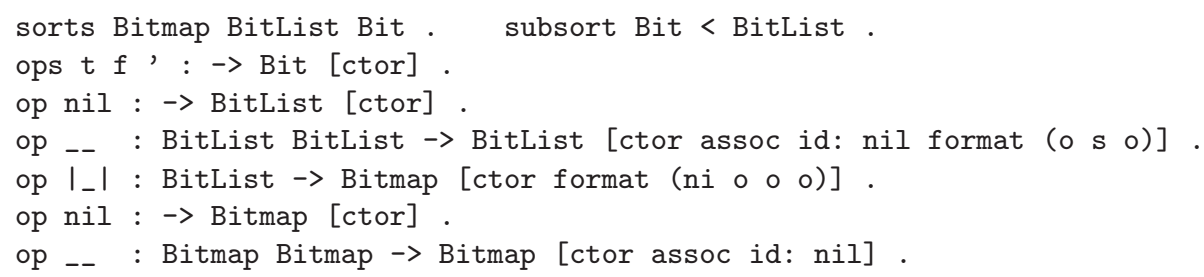

The location of each bit is computed from the location of the node which is the center of the bitmap. A function updateBitmap updates a node's bitmap when the node receives a power-on message (see rule recPower0n1) by setting each bit within the sensing range of the sender to $t$. The node then also checks whether its (updated) bitmap is completely covered by its neighbors. This is done by the function coverageAreaCovered, which returns false if some bit is ' $\mathrm{f}$ ' and returns true otherwise (owise):

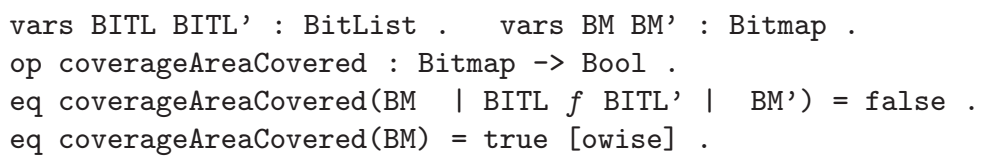

We choose to have 1 meter between each bit in a bitmap, which results in bitmaps with 400 bits (including the' bits) since the sensing range of a node is 10 meters.

\subsection{The Definition of Sensor Node Objects}

We model sensor nodes as objects of the class WSNode. A sensor node does not have an explicit identifier but can be identified by its location. We let locations be object identifiers by giving the subsort declaration subsort Location $<$ Oid .

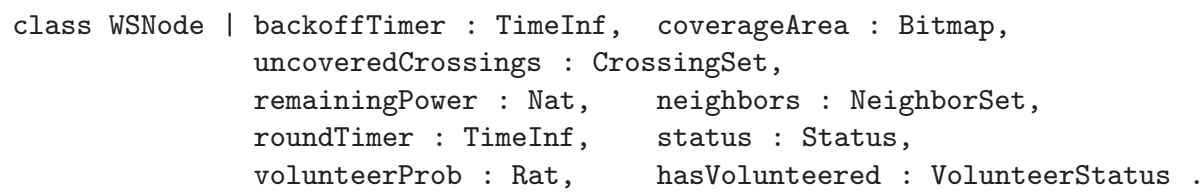

The attribute names are self-explanatory: backoffTimer denotes the time remaining until the node must perform an action; coverageArea contains the node's coverage area; remainingPower denotes the amount of power the node has left; roundTimer is the time remaining of the round; status denotes the node's status, which is either on, of $f$, or undecided; volunteerProb gives the probability for the node to volunteer as a starting node; and hasVolunteered denotes whether the node has volunteered as a starting node. 


\subsection{Modeling Time and Time Elapse}

We follow the guidelines in [17] for modeling time-dependent behaviors in objectoriented specifications. Time elapse is modeled by the tick rule

var C : Configuration. var $\mathrm{T}$ : Time.

crl $[$ tick $]:\{\mathrm{C}\} \Rightarrow\{\delta(\mathrm{C}, \mathrm{T})\}$ in time $\mathrm{T}$ if $\mathrm{T}<=\operatorname{mte}(\mathrm{C})$.

The function $\delta$ defines the effect of time elapse on a configuration, and the function mte defines the maximum amount of time that can elapse before some action must take place. These functions distribute over the objects and messages in a configuration and must be defined for single objects. The tick rule advances time nondeterministically by any amount $\mathrm{T}$ less than or equal to mte(C). Before executing the system, a time sampling strategy guiding the application of the tick rule must be defined (see Section 5.1). We import the built-in module NAT-TIME-DOMAIN-WITH-INF, which defines the time domain Time to be the natural numbers, with an additional constant INF (for $\infty$ ) of a supersort TimeInf.

The function $\delta$ is defined on a WSNode object by decreasing its timers and amount of remaining power according to the time that has elapsed:

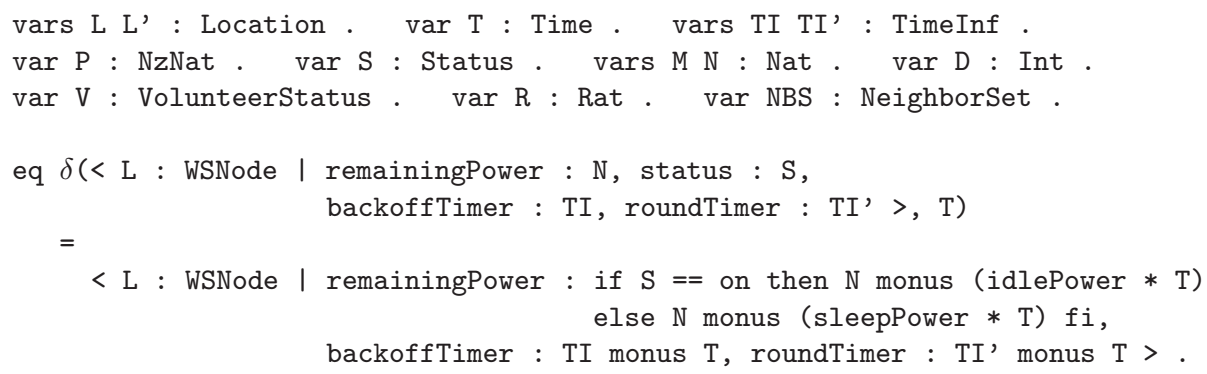

The constants idlePower and sleepPower denote the amount of power the node consumes per time unit (millisecond) when the node is active and inactive, respectively. The function monus is defined by $x$ monus $y=\max (0, x-y)$.

The function mte is defined so that time cannot advance when a node is in its volunteering process (undecided) - forcing the node to enter this process at the start of each round - and otherwise cannot advance beyond the expiration time of a timer, or beyond the time when the node would run out of power:

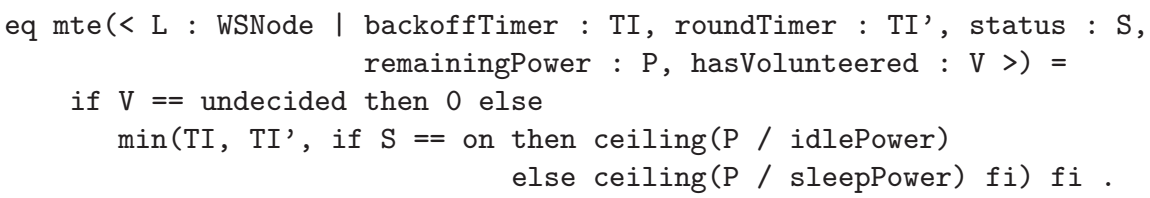

\subsection{Modeling Communication}

The informal description of the OGDC algorithm says that nodes broadcast messages within the radio range. Furthermore, a node does not know its neighbors. 
Most time related parameters in OGDC are set according to the transmission time of a message, which is assumed to be the same for all broadcast transmissions. This is a clear indication that transmission delays must be captured in the model. In 19 we show how such "area broadcast" with transmission delay $\Delta$ can be easily modeled in Real-Time Maude. The idea is that the sender $l$ sends a "broadcast message" broadcast $m$ from $l$, where $m$ is the message content, into the configuration. This broadcast message is then defined to be equivalent to a set of single messages dly (msg $m$ from $l$ to $l^{\prime}, \Delta$ ), one such message for each sensor node $l^{\prime}$ within the radio range of $l$.

Since the description of OGDC does not discuss packet collisions, and only mentions that OGDC also should work in the presence of message losses, we have not modeled problems that are due to packet collisions.

\subsection{Probabilistic Behaviors}

The OGDC algorithm exhibits probabilistic behaviors in that (i) some actions are performed with probability $p$, and (ii) some values are supposed to be set to "random values, drawn from a uniform distribution ..." As mentioned, Real-Time Maude does not provide explicit support for specifying probabilistic behavior. Instead, for simulation purposes, we define a function random, which generates a sequence of numbers pseudo-randomly and which satisfies Knuth's criteria for a "good" random number generator [7. The state must then contain an object of a class RandomNGen with an attribute seed which stores the ever-changing "seed" for random. Probabilistic behaviors can then be modeled by "sampling" a value from the given interval using the random function. For the purpose of specifying all possible behaviors, we could have-but have not, due to the resulting large reachable state spaces that would have made exhaustive analysis unfeasible - modeled probabilistic behavior by nondeterministic behavior by (i) letting a probabilistic action be enabled as long as the probability of it being performed is greater than 0 , and (ii) by letting the "random" value be a new variable, only occurring in the right-hand side of the rewrite rule, which can be given any value in the desired interval.

\subsection{Defining the Dynamic Behavior of the OGDC Algorithm}

The dynamic behavior of the OGDC algorithm is modeled in Real-Time Maude by 11 rewrite rules, 3 of which are given below.

At the start of each round of the OGDC algorithm, each node is in state undecided and must decide whether or not to volunteer as a starting node. This part of the protocol is described as follows in [22]:

A node volunteers to be a starting node with probability $p$ if its power exceeds a pre-determined threshold $P_{t}$. [...] If a sensor node volunteers, it sets a backoff timer to $\tau_{1}$ seconds, where $\tau_{1}$ is uniformly distributed in $\left[0, T_{d}\right]$. When the timer expires, the node changes its state to "ON", and broadcasts a power-on message. [...] The power-on message sent by the starting node contains (i) the 
position of the sender and (ii) the direction $\alpha$ along which the second working node should be located. This direction is randomly generated from a uniform distribution in $[0,2 \pi]$. [...] If the node does not volunteer itself to be a starting node, it sets a timer of $T_{s}$ seconds. [...]

This part of the OGDC algorithm is probabilistic, since a node decides to volunteer with probability $p$. We simulate such probabilistic behavior in the following rewrite rules by checking whether the next pseudo-random number generated in the system, modified to a value between 0 and 999 (randomProb (M), defined as random(M) rem 1000), is less than $R$, where $R$ denotes the current volunteering probability multiplied by 1000. The first rule models the start of the "starting node selection" phase when the node's hasVolunteered attribute is undecided:

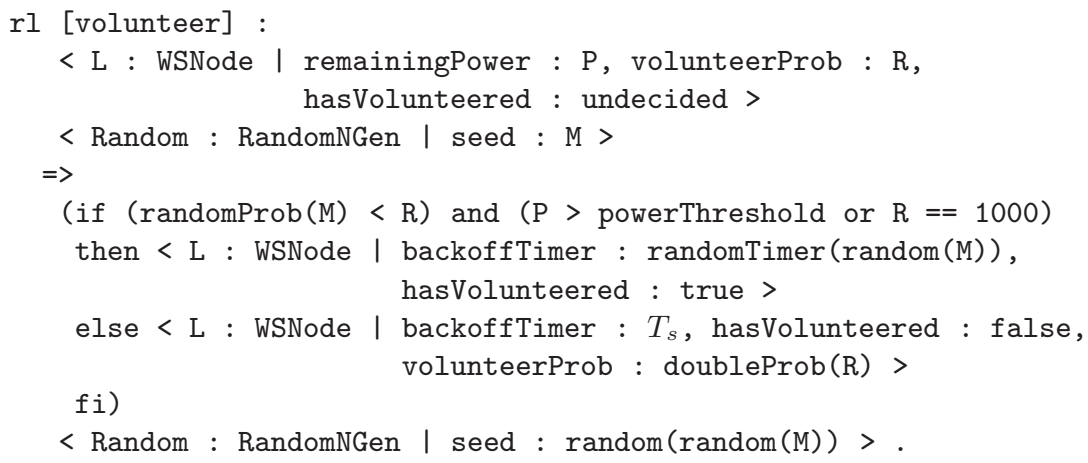

< Random : RandomNGen | seed : $\operatorname{random}(\operatorname{random}(\mathrm{M}))>$.

The node must also have sufficient remaining power $(\mathrm{P}>$ powerThreshold), or its volunteer probability must have reached $1(\mathrm{R}==1000)$. If the node volunteers, it sets its backoff timer to a random value between 0 and $T_{d}$ by the function randomTimer. If the node does not volunteer, it sets its backoff timer to $T_{s}$. The seed is also updated, so that the next application of this (or any other) rule will draw a completely different random number.

A node becomes active when its backoff timer expires. If the node volunteered as a starting node, it broadcasts a power-on message that contains the node's location and a random direction:

rl [startingNodePowerOn] :

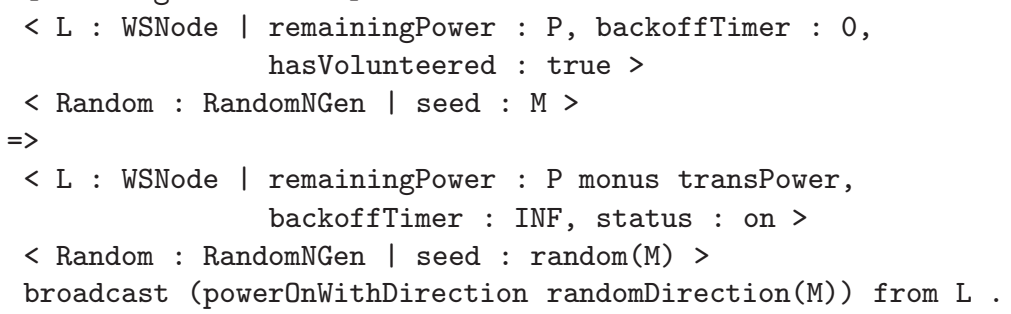

The node consumes transPower amount of power when it broadcasts a message.

The actions taken when a node receives a power-on message are described as follows in 22]: 
When a sensor node receives a power-on message, if the node is already "ON", or it is more than $2 r_{s}$ away from the sender node, it ignores the message; otherwise it adds this node to its neighbor list, and checks whether or not all its neighbors' coverage disks completely cover its own coverage disk. If so, the node sets its state to "OFF" and turns itself off. Otherwise [...]

The next rule models the case where the receiver has status undecided and its coverage area becomes entirely covered by its active neighbors (including the sender of the current power-on message). In this case, the node turns itself off:

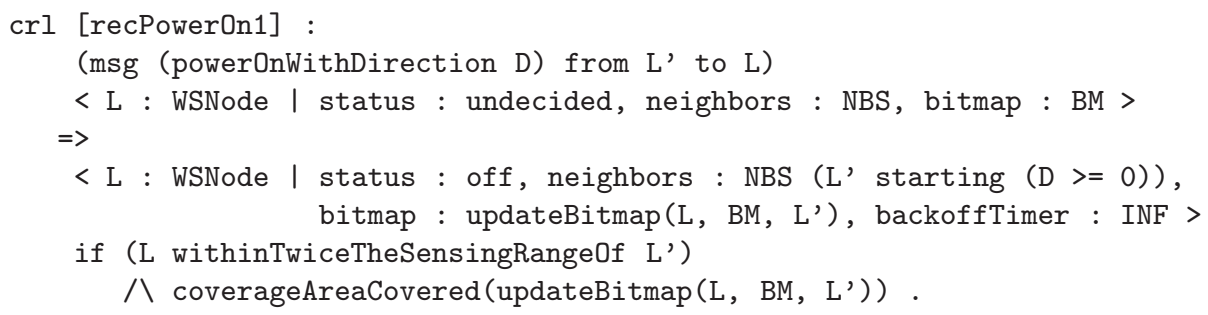

\section{Simulation and Formal Analysis of OGDC}

This section describes how the OGDC algorithm can be subjected to the following kinds of formal analysis in Real-Time Maude:

1. Monte Carlo simulation, with probabilistic behavior simulated using our pseudo-random number generator, by timed fair rewriting. In particular, we show how Real-Time Maude can perform all the simulations done by Zhang and Hou on the wireless extension of the network simulation tool ns-2.

2. Time-bounded reachability analysis and temporal logic model checking of all possible behaviors from some initial state with respect to the particular values generated by the pseudo-random generator. That is, our analysis is incomplete since we do not analyze all possible behaviors for a given network topology, but only those that can take place with the specific choice of pseudo-random numbers used to simulate the probabilistic behavior. Nevertheless, such analysis covers many different behaviors from a given state.

In our experiments, we use the same values for parameters such as sensing range $(10 \mathrm{~m})$, length of a round (1000 seconds), power consumption, transmission times, etc., as in the ns-2 simulations in [22]. In those simulations, 100 to 1000 nodes were "uniformly randomly distributed" in a $50 \mathrm{~m} \times 50 \mathrm{~m}$ sensing area.

\subsection{Defining Initial States and the Time Sampling Strategy}

To easily simulate large sensor networks with different node locations and initial seeds, we define a function genInitConf to generate initial states. The term genInitConf $(n$, seed) defines a configuration with $n$ sensor nodes scattered at pseudo-random locations within the sensing area, as well as a RandomNGen object 
with starting seed computed from the initial seed seed. (An initial state must also add the operator $\left\{_{-}\right\}$.) We can therefore generate initial states with any number of nodes, and/or place them in different locations, by just changing the parameters $n$ and/or seed in genInitConf.

In the following definition, each generated sensor node location $x \cdot y$ will have $0 \leq x \leq \mathrm{Xsize}$ and $0 \leq y \leq \mathrm{Ysize}$ (since rem is the remainder function):

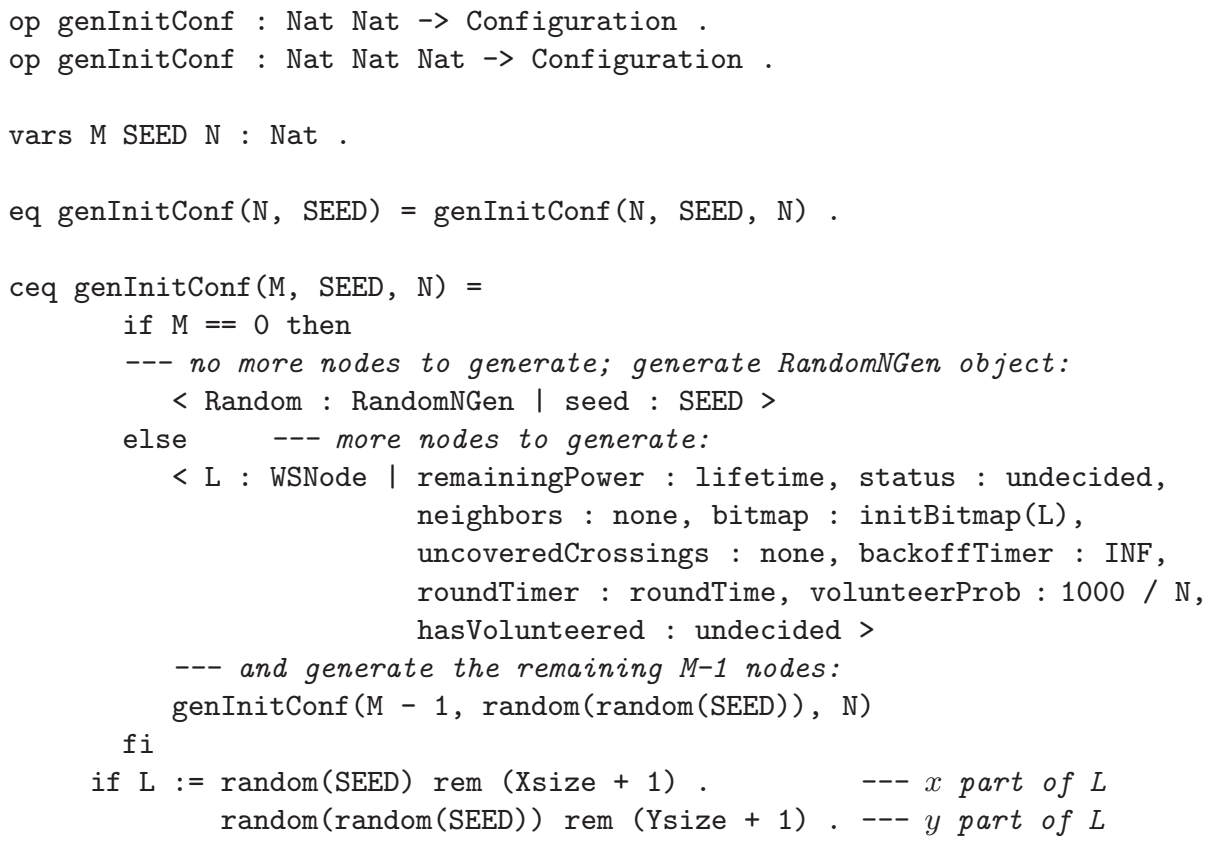

Each generated WSNode gets the appropriate initial values for its attributes. The third argument to the genInitConf in the main equation is needed to store the total number of nodes in the system (N) so that the volunteerProb attribute gets the correct initial value.

A time sampling strategy guiding the execution of the tick rule must be chosen before any analysis can take place. Since all events in the OGDC algorithm happen at specific times, we have shown in [16] that we can "fast forward" between these events without losing any interesting behaviors. Therefore, in our analysis, we use the maximal time sampling strategy declared by the Real-Time Maude command (set tick max def roundTime.) which advances time as much as possible, and corresponds to "event-driven simulation."

\subsection{The ns-2 Simulations of OGDC in Real-Time Maude}

In [22], Zhang and Hou use the network simulation tool ns-2 [12], with the wireless extension developed by the CMU Monarch group 4, to simulate the OGDC algorithm and measure the following essential performance metrics: 
- The number of active nodes and the percentage of sensing area coverage provided by those nodes at the end of the first round.

- The percentage of sensing area coverage and the total amount of remaining power for the whole system throughout the network's lifetime.

- The total time during which at least $\alpha$ percent of the sensing area is covered. (This can be done in the same way as the first two, and is not treated here.)

We cannot use Real-Time Maude's timed rewrite command directly to perform the corresponding analysis, since these performance metrics should be measured at different points in time throughout the lifetime of the system, and since the metrics themselves do not appear explicitly in the state. Therefore, we add to the initial state a record object that uses a timer to compute a performance metric at the same time (e.g., just before the end of the round) in each round during a simulation of the OGDC algorithm. The computed values are stored in an attribute of the record object as a list $n_{1}++n_{2}++\ldots++n_{k}$, where $n_{i}$ denotes the value of the metric at the end of round $i$. Given a sort NatList of lists of natural numbers, with concatenation operator _ ${ }^{++}+$and empty list nil, we can declare the record object class as follows:

class RecActNodes I activeNodes : NatList, timer : TimeInf . ops $r 1$ r2 r3 : -> Oid [ctor]. --- names of record objects

The following rule applies when the timer of the record object expires. It computes and stores the number of active nodes in the system, and resets the timer in order for it to be expire again at the same time in the next round:

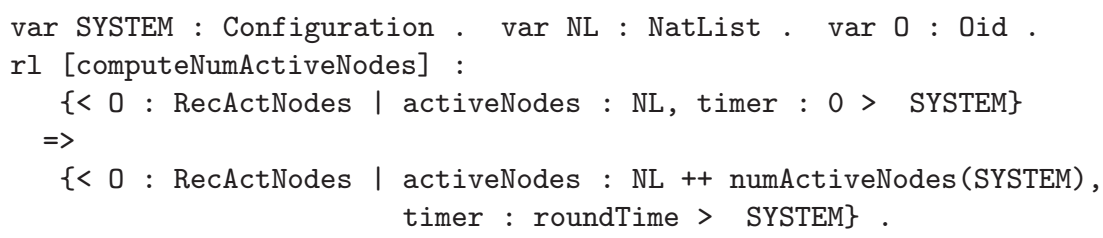

The function numActiveNodes computes the number of active nodes in a configuration. In the same way, we define record object classes RecCoverage\% and RecTotalPower, which compute, respectively, the percentage of the sensing area covered by the active nodes and the total amount of power in the system.

The first simulations in 22] investigate the number of active nodes and the percentage of coverage in the first round of the algorithm. The following timed fair rewrite command simulates a system with 600 nodes (in a $50 \mathrm{~m} \times 50 \mathrm{~m}$ sensing area) until the end of the first round of the protocol (in time < roundTime). The initial state contains two record objects, whose metrics will be computed when their timers expire just before the end of the first round (roundTime - 1): 
We have, unfortunately, not been able to get an answer to whether or not the ns-2 simulations in 22 actually took the transmission delays into account, although the second author told us it is quite likely that they did not. Therefore, we have also performed the simulations without transmission delays (by just removing the dly-part from the single messages created by the broadcast). The following table shows the results of the ns-2 simulations, as well as of the RealTime Maude simulations both with and without transmission delays, for finding the number of active nodes at the end of the first round for 200, 400, and 600 nodes in the same $50 m \times 50 m$ area. For the Real-Time Maude simulations, each number represents the average result of five simulations, obtained by using five different initial seeds (and hence getting five different placements of the nodes):

\begin{tabular}{l|c|c|c|} 
Number of nodes in sensing area & 200 & 400 & 600 \\
\hline \# active nodes in ns-2 simulations & 17 & 18 & 18 \\
\hline \# active nodes in Real-Time Maude simulations with trans. delay & 34 & 45 & 55 \\
\hline \# active nodes in Real-Time Maude simulations without trans. delay & 21 & 22 & 22 \\
\hline
\end{tabular}

Indeed, the results of the Real-Time Maude simulations that ignore transmission delays are quite similar to the results of the ns- 2 simulations. It is therefore tempting to conjecture that our Real-Time Maude simulations with transmission delays give a reasonably accurate estimate of the performance of OGDC in such a setting. In that case, one can conclude that the results of ns- 2 simulations are actually quite misleading and that our formal model provides a more accurate simulation setting for OGDC than ns-2 with the wireless extension.

\subsection{Further Real-Time Maude Analysis of the OGDC Algorithm}

We give some examples of how we can further formally analyze correctness and worst-case performance of the OGDC algorithm by using Real-Time Maude's search and model checking capabilities. Due to the large states involved, we restrict such analyses to systems with 5 to 6 nodes (in a $25 m \times 25 m$ area), which is much fewer nodes than in a real WSN. Nevertheless, exhaustive analysis with 3 to 4 nodes has uncovered subtle bugs in cryptographic protocols [9] and other kinds of network protocols (e.g., [18]).

The following find latest command finds the latest possible time the network enters the steady state phase (such that steadyStatePhase (...)), and thereby also finds out whether this phase is always reached in the first round.

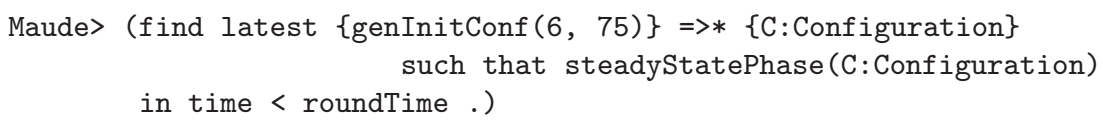


That is, the system will reach the steady state phase in at most $372 \mathrm{~ms}$. One round of the OGDC algorithm is 1000 seconds, which means that the network spends most of its lifetime performing its sensing task.

Another correctness requirement is that the network stays in the steady state phase throughout the first round, once this phase has been reached. We use RealTime Maude's temporal logic model checker, and define an atomic proposition steady-state to hold when the network is in steady state phase:

op steady-state : -> Prop [ctor].

eq $\{\mathrm{C}\} \quad$ I= steady-state = steadyStatePhase $(\mathrm{C})$.

The following command checks whether all states following a state in the steady state phase are also in this phase $(A \Rightarrow B$ is an abbreviation for [] ( $A$ $->B)$.

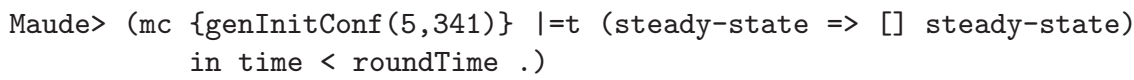

Result Bool : true

The most important correctness criterion is that the entire sensing area is covered by the active nodes when the system is in the steady state phase (and all nodes together cover the entire area and each node has power to last to the end of the round). The following command searches for a state which is in steady state but where the entire $20 m \times 20 m$ sensingArea is not covered by the active nodes:

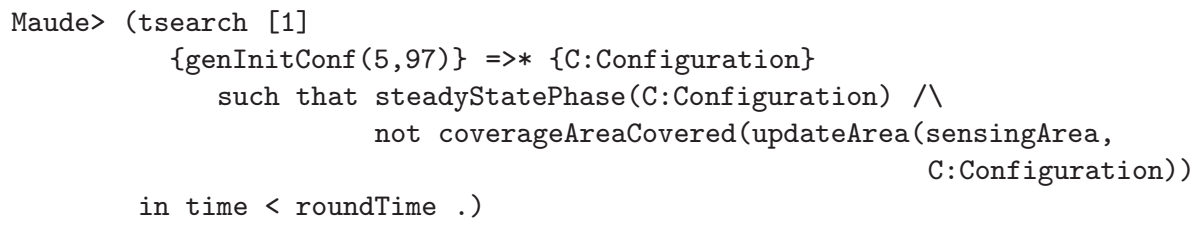

The function updateArea updates the bitmap by changing bits that are covered by the active nodes in C to t. The command returned 'No solution.'

Performance figures. The following table shows, for each command presented in this paper, and for the above search command with a different topology (given by seed 1) which does not cover the sensing area: the number of sensor nodes; execution time; and memory usage when executed on a 3.6 GHz Intel Xeon:

\begin{tabular}{|c|c|c|c|c|c|c|c|}
\hline tfrew 1 rd & tfrew 1 rd & tfrew 1 rd & tfrew 50 rds & find & mc & tsearch & tsearch \\
\hline 200 & 400 & 600 & 200 & 6 & 5 & $6, \mathrm{~s}=1$ & $5, \mathrm{~s}=7197$ \\
\hline $180 \mathrm{sec}$ & $1243 \mathrm{sec}$ & $5034 \mathrm{sec}$ & $4931 \mathrm{sec}$ & $4187 \mathrm{sec}$ & $26 \mathrm{sec}$ & $679 \mathrm{sec}$ & $227 \mathrm{sec}$ \\
\hline $85 \mathrm{MB}$ & $100 \mathrm{MB}$ & $112 \mathrm{MB}$ & $93 \mathrm{MB}$ & $525 \mathrm{MB}$ & $147 \mathrm{MB}$ & $1.3 \mathrm{~GB}$ & $430 \mathrm{MB}$ \\
\hline
\end{tabular}

The paper 22 does not mention the performance of their ns-2 simulations. 


\section{Concluding Remarks}

Wireless sensor networks are a new kind of network whose modeling, simulation, and/or analysis pose a set of challenges to both network simulation tools and formal tools. OGDC is a state-of-the-art WSN algorithm where new forms of communication and advanced data types must be captured at an appropriate level of abstraction. In this paper we have shown how OGDC was formally specified, simulated, and analyzed using Real-Time Maude. To the best of our knowledge, this is the first formal analysis of an advanced WSN algorithm. Our formal specification captures the behavior of the algorithm at a high level of abstraction and - being precise, intuitive, and operational-could make a good starting point for an implementation of the OGDC algorithm on sensor networks.

We could measure all performance metrics measured in the ns- 2 simulations in 22 during our "Monte Carlo" simulations. Our simulations showed significantly worse performance of the OGDC algorithm than the ns- 2 simulations. Trying to understand why - unlike in the ns-2 simulations - we got more active nodes when more nodes were deployed in the same sensing area, we found that the "tie-breaking" mechanism in OGDC does not break many ties when transmission delays are taken into account. To check this hypothesis, we also simulated OGDC in Real-Time Maude in a setting without transmission delays, and got results that were similar to the ns- 2 results. It is therefore quite likely that our simulations, which take the delays into account, provide much more accurate performance estimates than the ns- 2 simulations that may have ignored such delays. Furthermore, based on communication with Jennifer Hou, it seems that developing the Real-Time Maude specification and performing the RealTime Maude analysis required much less effort than using a specialized network simulation tool to analyze OGDC.

Our work should continue in different directions. First, we focus on simplicity and elegance when modeling coverage areas and defining functions on such areas. There is a price to pay for this when we have hundreds of nodes, each with a bitmap with 400 "bits." Therefore, more efficient representations of coverage areas should be developed. This would enable us to perform search and model checking on larger networks.

Second, we have not modeled probabilistic behaviors as such, but have used a "sampling" technique for simulation purposes. This means that we cannot reason about probabilistic properties. We should therefore combine Real-Time Maude with methods and tools for probabilistic systems, such as PMaude [1, and should develop methods to fruitfully analyze probabilistic real-time specifications.

Finally, we should also capture message losses due to packet collisions.

Acknowledgments. We are grateful to Jennifer Hou for suggesting the OGDC algorithm as a challenging modeling task, and for discussions on sensor networks, to José Meseguer for discussions on modeling communication in sensor networks, and to the anonymous reviewers for helpful comments on earlier versions of this paper. Support by the Research Council of Norway is also gratefully acknowledged. 


\section{References}

1. Agha, G., Meseguer, J., Sen, K.: PMaude: Rewrite-based specification language for probabilistic object systems. In: Proc. QAPL'05 (2005)

2. Akyildiz, I.F., Su, W., Sankarasubramaniam, Y., Cayirci, E.: Wireless sensor networks: A survey. Computer Networks 38, 393-422 (2002)

3. Clavel, M., Dúran, F., Eker, S., Lincoln, P., Martí-Oliet, N., Meseguer, J., Talcott, C.: Maude Manual (Version 2.2), December 2005. http://maude.cs.uiuc.edu (2005)

4. CMU monarch extensions to ns. http://www.monarch.cs.cmu.edu/

5. Coleri, S., Ergen, M., Koo, T.J.: Lifetime analysis of a sensor network with hybrid automata modelling. In: WSNA '02, ACM Press, New York (2002)

6. Kim, M., Dutt, N., Venkatasubramanian, N.: Policy construction and validation for energy minimization in cross layered systems: A formal method approach. In: IEEE RTAS'06 Work-in-Progress Session, pp. 25-28. IEEE Computer Society Press, Los Alamitos (2006)

7. Knuth, D.E.: The Art of Computer Programming: Seminumerical Algorithms, 2nd edn., vol. 2. Addison-Wesley, London, UK (1981)

8. Lien, E.: Formal modelling and analysis of the NORM multicast protocol using Real-Time Maude. Master's thesis, Dept. of Linguistics, University of Oslo (2004)

9. Lowe, G.: An attack on the Needham-Schroeder public-key authentication protocol. Information Processing Letters 56, 131-133 (1995)

10. Meseguer, J.: Membership algebra as a logical framework for equational specification. In: Parisi-Presicce, F. (ed.) WADT 1997. LNCS, vol. 1376, Springer, Heidelberg (1998)

11. Nair, S., Cardell-Oliver, R.: Formal specification and analysis of performance variation in sensor network diffusion protocols. In: MSWiM '04, ACM Press, New York (2004)

12. ns-2 network simulator. http://www.isi.edu/nsnam/ns

13. Ölveczky, P.C., Caccamo, M.: Formal simulation and analysis of the CASH scheduling algorithm in Real-Time Maude. In: Baresi, L., Heckel, R. (eds.) FASE 2006 and ETAPS 2006. LNCS, vol. 3922, pp. 357-372. Springer, Heidelberg (2006)

14. Ölveczky, P.C., Meseguer, J.: Specification of real-time and hybrid systems in rewriting logic. Theoretical Computer Science 285, 359-405 (2002)

15. Ölveczky, P.C., Meseguer, J.: Specification and analysis of real-time systems using Real-Time Maude. In: Wermelinger, M., Margaria-Steffen, T. (eds.) FASE 2004. LNCS, vol. 2984, Springer, Heidelberg (2004)

16. Ölveczky, P.C., Meseguer, J.: Abstraction and completeness for Real-Time Maude. In: Proc. WRLA'06 (2006)

17. Ölveczky, P.C., Meseguer, J.: Semantics and pragmatics of Real-Time Maude. Higher-Order and Symbolic Computation, To appear (2007)

18. Ölveczky, P.C., Meseguer, J., Talcott, C.L.: Specification and analysis of the AER/NCA active network protocol suite in Real-Time Maude. Formal Methods in System Design 29, 253-293 (2006)

19. Ölveczky, P.C., Thorvaldsen, S.: Formal modeling and analysis of wireless sensor network algorithms in Real-Time Maude. In: IPDPS 2006, IEEE, NJ, New York (2006) 
140 P.C. Ölveczky and S. Thorvaldsen

20. Rodríguez, D.E.: On modelling sensor networks in Maude. In: Proc. WRLA 2006 (2006)

21. Thorvaldsen, S., Ölveczky, P.C.: Formal modeling and analysis of the OGDC wireless sensor network algorithm in Real-Time Maude. Manuscript. http://www.ifi.uio.no/RealTimeMaude/OGDC (Oct. 2005)

22. Zhang, H., Hou, J.C.: Maintaining sensing coverage and connectivity in large sensor networks. Wireless Ad Hoc and Sensor Networks: An International Journal, vol. 1 (2005) 